\title{
4 A novel regulatory relationship between RIPK4 and ELF3 in keratinocytes
}

5

6 Glen M. Scholz, Nur S. Sulaiman ${ }^{1}$, Sahar Al Baiiaty ${ }^{1}$, Mei Qi Kwa ${ }^{2}$, and Eric C. Reynolds 7

8 Oral Health Cooperative Research Centre, Melbourne Dental School, Bio21 Molecular

9 Science and Biotechnology Institute, University of Melbourne, Victoria 3010, Australia.

$11{ }^{1}$ These authors made equal contributions.

$12{ }^{2}$ Current address: Institute of Molecular and Cell Biology, A*STAR, Singapore.

14 Running title: RIPK4 and ELF3 in keratinocytes

16 The abbreviations used are: ActD, actinomycin D; CHX, cycloheximide; ELF3, E74-like

17 factor 3; GRHL3, Grainyhead-like 3; IRF6, Interferon regulatory factor 6; IVL, involucrin;

18 OVOL1, Ovo-like zinc-finger 1; RIPK, Receptor-interacting protein kinase; SPRR, small

19 proline-rich protein; TGM, transglutaminase. 


\section{1 \\ Abstract}

Keratinocytes are central to the barrier functions of surface epithelia, such as the gingiva and epidermis. RIPK4 is a key regulator of keratinocyte differentiation; however, the signalling pathways in which it functions remain poorly defined. In this study, we identified a regulatory relationship between RIPK4 and ELF3, an ETS family transcription factor. RIPK4 was shown to be important for the upregulation of ELF3 gene expression by the PKC agonist PMA in both oral and epidermal keratinocytes. RIPK4 promotes keratinocyte differentiation in part by phosphorylating and thereby activating the IRF6 transcription factor. Significantly, silencing of IRF6 inhibited the PMA-inducible expression of ELF3. A role for the GRHL3 transcription factor, a downstream target gene of IRF6, in the regulation of ELF3 expression was similarly demonstrated. ELF3 has previously been shown to regulate the expression of SPPR1A and SPRR1B, small proline-rich proteins that contribute to the cornification of keratinocytes. Consistently, RIPK4 and IRF6 were shown to be required for the PMAinducible expression of SPRR1A and SPRR1B. They were also shown to be important for the upregulation of TGM1, a transglutaminase that catalyses the cross-linking of proteins, including small proline-rich proteins, during keratinocyte cornification. RIPK4 was also shown to upregulate the expression of TGM2 independently of IRF6. Collectively, our findings position RIPK4 upstream of a hierarchal IRF6-GRHL3-ELF3 transcription factor pathway in keratinocytes, as well as provide insight into a potential role for RIPK4 in the regulation of keratinocyte cornification. 


\section{Introduction}

The stratified squamous epithelia of the oral cavity, as well as other surface epithelia (e.g. epidermis), provide protection against mechanical and chemical damage, and biological insults $[1,2]$. The epithelia, which are organised into layers of morphologically and biochemically distinct cells, are highly dynamic and maintained through tightly regulated keratinocyte proliferation and differentiation [2,3]. Tissue renewal, in turn, is initiated by stem cell populations in the basal layer that undergo a limited number of cell divisions before initiating terminal differentiation as they migrate towards the superficial layers. Depending on anatomical location, keratinocytes may also become enucleated, flattened, and cornified [2-4].

Cornification greatly strengthens the barrier functions of keratinocytes. During the final stages of keratinocyte terminal differentiation, the nucleus and its DNA are degraded and keratin filaments are aggregated into tight bundles by filaggrin to promote the collapsing of the cell into a flattened shape $[2,4]$. Concomitantly, the cornified envelope is assembled just under the cell membrane through the cross-linking of various proteins (e.g. involucrin, loricrin, and small proline-rich proteins) by calcium-dependent transglutaminases, for example, transglutaminase-1 (TGM1). Intracellular lipids from lamellar bodies are also deposited in the cell membrane, where they become covalently attached to the cornified envelope as well as extruded from the cell to form intercellular lamellae. Collectively, this results in the replacement of the keratinocyte cell membrane with an insoluble structure that protects the underlying epithelial tissues $[2,4]$. Keratinocytes also play active roles in epithelial homeostasis and host defence by producing cytokines that promote inflammation and wound healing in response to injury and infection [5].

Receptor-interacting protein kinase 4 (RIPK4) is an important regulator of keratinocyte differentiation [6]. For instance, the epidermis of Ripk4-deficient mice is disorganised and expanded, and the outermost cornified layers are absent, resulting in defective barrier function 
$[6,7]$. Mutations in RIPK4 cause Bartsocas-Papas syndrome [8, 9], a congenital syndrome

67 that is characterised by severe oral and epidermal abnormalities. At the molecular level, 68 RIPK4 can activate NF- $\mathrm{B}$ [10-13], a critical regulator of epithelial tissue homeostasis [14].

69 Significantly, RIPK4 can directly activate Interferon regulatory factor 6 (IRF6) [12]. IRF6 is 70 an important transcriptional regulator of keratinocyte differentiation and promotes the switch 71 from proliferation to differentiation $[15,16]$. IRF6 regulates keratinocyte differentiation in 72 part by inducing the expression of the transcription factors Grainyhead-like 3 (GRHL3) and 73 Ovo-like zinc-finger 1 (OVOL1) [12, 17, 18]. Similar to Ripk4-deficient mice, the spinous layer in the epidermis of Irf6-deficient mice is expanded, and the granular and cornified layers appear to be absent [15]. We recently established that RIPK4 also regulates the expression of proinflammatory cytokines by keratinocytes through its activation of IRF6 [19]. Thus, RIPK4 appears to function as a key regulatory nodal point in the maintenance of epithelia homeostasis.

To understand further the role of RIPK4 in keratinocytes, we sought to identify additional target genes of RIPK4 signalling. We show here that RIPK4 signalling regulates the expression of the ETS family transcription factor E74-like factor 3 (ELF3) in human keratinocytes. Specifically, our data suggest that RIPK4 promotes ELF3 gene expression via the IRF6-mediated upregulation of GRHL3. Moreover, this RIPK4-regulated IRF6-GRHL3ELF3 transcriptional network appears to control the expression of genes (e.g. SPRR1 and TGM1) that directly mediate keratinocyte cornification.

\section{Materials and Methods}

\subsection{Reagents}

Keratinocyte serum-free medium and supplements (human EGF and bovine pituitary extract) (Cat. no. 37010022), GlutaMax-1 (Cat. no. 35050061), Opti-MEM I reduced serum medium 
91 (Cat. no. 31985062), Lipofectamine RNAiMAX transfection reagent (Cat. no. 13778150), and

92 the Silencer Select RIPK4 siRNA (Cat. no. 4390824, siRNA ID: s28865) and GRHL3 siRNA

\subsection{Stimulation of keratinocytes with phorbol 12-myristate 13-acetate}

OKF6/TERT-2 cells and normal human epidermal keratinocytes were allowed to adhere overnight. Thereafter, the cells were stimulated with $100 \mathrm{ng} / \mathrm{ml}$ phorbol 12-myristate 13 acetate (PMA) in dimethylsulfoxide (DMSO) for the times indicated in the figure legends. Time-matched, control cells were treated with $0.1 \%$ DMSO.

\subsection{Inhibition of RNA transcription and protein synthesis}

RNA transcription was inhibited by pretreating OKF6/TERT-2 cells with $1 \mu \mathrm{g} / \mathrm{ml}$ actinomycin D for 30 min prior to stimulation with PMA for $6 \mathrm{~h}$. Protein synthesis was 
inhibited by pretreating cells with $20 \mu \mathrm{g} / \mathrm{ml}$ cycloheximide for $30 \mathrm{~min}$ prior to stimulation

with PMA for $6 \mathrm{~h}$. Time-matched, control cells were treated with $0.1 \%$ DMSO or $0.1 \%$ ethanol, respectively.

\subsection{RNA interference-mediated gene silencing}

A reverse-transfection protocol was used for siRNA transfection of OKF6/TERT-2 cells and normal human epidermal keratinocytes. Briefly, the siRNA were diluted to $120 \mathrm{nM}$ with 100 $\mu 1$ Opti-MEM I reduced serum medium, mixed with $100 \mu$ l Opti-MEM I reduced serum medium containing $1 \mu 1$ Lipofectamine RNAiMAX (Life Technologies), and incubated at room-temperature for 15-20 min. OKF6/TERT-2 cells and normal human epidermal keratinocytes $\left(2 \times 10^{5}\right.$ cells in $1 \mathrm{ml}$ medium) were seeded into 12-well plates and incubated with the siRNA transfection cocktail overnight. Thereafter, the medium was replaced and the cells stimulated with PMA 48 h (OKF6/TERT-2 cells) or 72 h (normal human epidermal keratinocytes) post-transfection.

\subsection{RNA purification and reverse transcription}

Total RNA was purified using the ReliaPrep RNA Cell miniprep system (Promega, Cat. no. 133 Z6012), which includes an on-column DNase-treatment step. RNA was reverse transcribed 134 into cDNA using random primers and GoScript Reverse Transcriptase (Promega, Cat. no. 135 A6110) according to the manufacturer's instructions. Briefly, 500 ng RNA was incubated in $13620 \mu \mathrm{l}$ of reaction buffer supplemented with $\mathrm{MgCl}_{2}$, PCR nucleotide mix, RNasin ribonuclease 137 inhibitor, $500 \mathrm{ng}$ random primers, and $1 \mu \mathrm{l} \mathrm{GoScript} \mathrm{Reverse} \mathrm{Transcriptase,} \mathrm{for:} 5$ min at $13825^{\circ} \mathrm{C}, 60 \mathrm{~min}$ at $42^{\circ} \mathrm{C}$, and $15 \mathrm{~min}$ at $75^{\circ} \mathrm{C}$. The cDNA was typically diluted $1: 4$ with water 139 prior to analysis by real-time PCR. 


\subsection{Gene expression profiling}

Two micrograms of RNA was reverse transcribed as described above. The cDNA $(100 \mathrm{ng} / \mu \mathrm{l})$ was mixed with TaqMan OpenArray Real-Time master mix (Life Technologies, Cat no.

4462164), and then loaded onto an OpenArray Human Inflammation plate (Life

Technologies, Cat. no. 4475389) using an OpenArray AccuFill System. PCR was performed on a QuantStudio 12K Flex Real-Time PCR System. The data were normalised against hypoxanthine-guanine phosphoribosyltransferase (HPRT) gene expression using Expression Suite Software (version 1.0.1).

\subsection{Quantitative real-time PCR}

Quantitative real-time PCR (qPCR) was performed in triplicate using GoTaq Probe qPCR

Master Mix (Promega, Cat. no. A6102) and pre-developed TaqMan assays (Life

Technologies) for the following genes: ELF3 (Assay ID: Hs00963881_m1), GRHL3 (Assay

ID: Hs00297962_m1), IRF6 (Assay ID: Hs00196213_m1), IVL (Assay ID:

Hs00902520_m1), OVOL1 (Assay ID: Hs00190060_m1), RIPK4 (Assay ID:

Hs01062501_m1), SPRR1A (Assay ID: Hs00954595_s1), SPRR1B (Assay ID:

Hs00824893_m1), TGM1 (Assay ID: Hs00165929_m1), TGM2 (Assay ID:

Hs00190278_m1), and TGM3 (Assay ID: Hs00162752_m1). PCR was performed on a

QuantStudio 7 Flex Real-Time PCR System, and the data was normalised against HPRT (Life

Technologies, Cat. no. 4326321E) or TBP (Life Technologies, Cat. no. 4326322E) gene expression.

\subsection{Statistical analysis}

Data combined from three or more independent biological replicates are presented as the mean \pm SEM. Statistical analysis was performed using GraphPad Prism 6 (GraphPad 
Software). Differences between two groups were evaluated using the Student's $t$ test. For multiple comparisons, statistical analysis was performed using a one-way analysis of variance (ANOVA). A $p$ value $<0.05$ was considered to be statistically significant.

\section{Results}

\subsection{RIPK4 regulates ELF3 gene expression in human keratinocytes}

In addition to regulating the PKC-mediated differentiation of keratinocytes [12], RIPK4 also regulates their expression of proinflammatory cytokines [19]. To understand further the function of RIPK4 in keratinocytes, we sought to identify additional target genes of RIPK4 signalling. To that end, OKF6/TERT-2 human oral keratinocytes (hereafter referred to as OKF6 cells) were transfected with a RIPK4 siRNA (Fig. 1A), and subsequently stimulated with the PKC agonist phorbol 12-myristate 13-acetate (PMA). Genes that were dependent on RIPK4 for their PMA-inducible expression were identified using the OpenArray Human Inflammation panel, and specific genes then validated by real-time PCR (qPCR). In addition to inflammatory cytokines, including some we had recently identified (e.g. CCL5) [19], ELF3 was identified as a PMA-inducible, RIPK4-dependent gene (Fig. 1B). ELF3 is an epitheliumspecific ETS family transcription factor, and its expression is upregulated during calciuminduced epidermal keratinocyte differentiation [20, 21]. Basal ELF3 gene expression in OKF6 cells was also found to be RIPK4-dependent (Fig. 1B). Given that RIPK4 partly regulates downstream gene expression by activating IRF6 [12], we investigated whether IRF6 was important for ELF3 gene expression. The transfection of OKF6 cells with an IRF6 siRNA, which reduced IRF6 expression by $>80 \%$ (Fig. 1C), strongly inhibited the PMA-inducible expression of ELF3 (Fig. 1D).The silencing of RIPK4 and IRF6 also inhibited the stimulation of ELF3 gene expression by PMA in normal human epidermal keratinocytes (Fig. 1E-F). 
190 Collectively, these data suggest that RIPK4 regulates ELF3 gene expression in keratinocytes by activating IRF6.

\subsection{ELF3 gene expression is regulated downstream of GRHL3 in keratinocytes}

A putative IRF6-response element has recently been described for several IRF6-regulated genes, including GRHL3 $[17,18]$. However, we were unable to identify potential binding sites for IRF6 in the ELF3 gene (data not shown). IRF6 promotes PKC-mediated keratinocyte differentiation in part by upregulating the expression of GRHL3 [12]. Therefore, we compared the kinetics of the PMA-inducible expression of ELF3 and GRHL3 in OKF6 cells. Significantly, ELF3 gene expression (Fig. 2A) was induced more slowly than GRHL3 (Fig. 2B). OKF6 cells were also pretreated with actinomycin D (ActD) prior to PMA stimulation to demonstrate that the increase in ELF3 mRNA levels was due to transcription and not mRNA stabilisation (Fig. 2C). Given these findings, we investigated if GRHL3 was important for ELF3 gene expression. Indeed, the silencing of GRHL3 in OKF6 cells inhibited the PMAinducible expression of ELF3 (Fig. 2D). The PMA-inducible expression of involucrin (IVL), an early marker of keratinocyte terminal differentiation, and a component of the cornified envelope [2, 4], was also inhibited (Fig. 2E). As expected, the PMA-inducible expression of OVOL1, a direct IRF6 target gene [17], was unaffected by the silencing of GRHL3 (Fig. 2F). Taken together, these results suggest that GRHL3 functions downstream of RIPK4 and IRF6 to regulate ELF3 gene expression in keratinocytes.

\subsection{Inhibition of protein synthesis upregulates ELF3 gene expression in keratinocytes}

212 To investigate further the mechanism underlying the PMA-inducible expression of ELF3 in 213 keratinocytes, the effect of the protein synthesis inhibitor cycloheximide (CHX) on ELF3 214 gene expression was investigated. As shown in Fig. 3A, CHX did not inhibit the PMA- 
inducible expression of ELF3. However, CHX treatment alone resulted in a largely

comparable increase in ELF3 mRNA levels (Fig. 3A). OKF6 cells were also treated with CHX and ActD concurrently to confirm that the increase in ELF3 mRNA was transcriptiondependent (Fig. 3A). In view of these findings, the effects of CHX on basal and PMAinducible IRF6 and GRHL3 gene expression were also investigated. As for ELF3, IRF6 and GRHL3 mRNA levels were significantly increased in CHX-treated cells (Fig. 3B-C).

Furthermore, the magnitudes of the stimulatory effects of CHX on IRF6 and GRHL3 mRNA levels were comparable to those exerted by PMA (Fig. 3B-C). A further small increase in IRF6 mRNA levels was apparent when the cells were treated with PMA and CHX concurrently (Fig. 3B). Notably, concurrent treatment with PMA and CHX synergistically increased GRHL3 mRNA levels (Fig. 3C). RIPK4 mRNA levels were also upregulated in response to $\mathrm{CHX}$ treatment (Fig. 3D). These data suggest that, in addition to positive regulation, the expression of ELF3, IRF6, GRHL3, and RIPK4 in oral keratinocytes (e.g. OKF6 cells) may also be negatively regulated by transcriptional repressor proteins.

\subsection{RIPK4 regulates SPRR1 gene expression in keratinocytes}

ELF3 has previously been shown to regulate the expression of small proline-rich proteins (SPRRs) [21-23], which are important components of the cornified envelope of terminally 233 differentiated keratinocytes [2, 4]. For example, SPRR1 is a major component of the cell 234 envelope of human oral keratinocytes [24]. Having established that RIPK4 regulates ELF3 235 gene expression (Fig. 1), we therefore wanted to determine the importance of RIPK4 for 236 SPRR1 gene expression. The stimulation of OKF6 cells with PMA resulted in the 237 upregulation of SPRR1A and SPRR1B gene expression, and this was inhibited by the 238 silencing of RIPK4 (Fig. 4A-B). RIPK4 was shown to also be important for the PMA239 inducible expression of SPRR1A and SPRR1B in epidermal keratinocytes (Fig. 4C-D). 

264

Similar to Ripk4-deficient mice, the cornified layers are also absent from the epidermis of Irf6-deficient mice [15]. Significantly, the PMA-inducible expression of SPRR1A and SPRR1B in OKF6 cells were shown to be IRF6-dependent (Fig. 4E-F). Interestingly, GRHL3 was important for the upregulation of SPRR1A gene expression (Fig. 4G), whereas the PMAinducible expression of SPRR1B was GRHL3-independent (Fig. 4H). The importance of the upregulation of ELF3 expression by PMA for subsequent SPRR1 gene induction was examined further by pretreating OKF6 cells with CHX. Consistently, the PMA-inducible expression of SPRR1A and SPRR1B were inhibited by CHX (Fig. 4I-J). In contrast to ELF3 basal gene expression (Fig. 3A), CHX did not increase the basal gene expression levels of either SPRR1A (Fig. 4I) or SPRR1B (Fig. 4J). Taken together, these results are consistent with RIPK4 regulating SPRR1A and SPRR1B expression in keratinocytes through the IRF6dependent induction of ELF3 gene expression.

\subsection{RIPK4 regulates TGM1 expression in keratinocytes}

The cross-linking of SPRRs to other proteins (e.g. loricrin) by transglutaminases (e.g. TGM1 and TGM3) increases the strength and flexibility of the cornified envelope [2, 4]. Therefore, the importance of RIPK4 for the expression of TGM1 and TGM3 in keratinocytes was investigated. The stimulation of OKF6 cells with PMA resulted in the RIPK4-dependent upregulation of TGM1 gene expression (Fig. 5A). The PMA-inducible expression of TGM1 in epidermal keratinocytes was also RIPK4-dependent (Fig. 5B). Similarly, the PMAinducible expression of TGM1 in OKF6 cells was also inhibited by the silencing of IRF6 (Fig. 5C) and GRHL3 (Fig. 5D). Given that the data presented in Fig. 3 suggested that ELF3 262 basal gene expression in OKF6 cells is partly regulated via transcriptional repression, the 263 effect of CHX on TGM1 gene expression was determined. CHX did not increase TGM1 mRNA levels in OKF6 cells (Fig. 5E), thereby distinguishing the mechanisms regulating 
265

1

TGM1 and ELF3 basal gene expression in OKF6 cells. In contrast to TGM1, TGM3 gene expression in OKF6 cells was not upregulated by PMA (data not shown). These data suggest that TGM1 gene expression in PMA-treated keratinocytes is controlled by a RIPK4-IRF6GRHL3 regulatory network.

\subsection{RIPK4 regulates TGM2 gene expression in keratinocytes independently of IRF6}

In contrast to TGM1 and TGM3, TGM2 is ubiquitously expressed and does not appear to play a role in keratinocyte cornification [25]. However, extracellular TGM2 can bind and crosslink extracellular matrix (ECM) components, which may help stabilise ECM-cell interactions [25]. Therefore, we investigated whether RIPK4 can regulate TGM2 gene expression. As shown in Fig. 6A, TGM2 mRNA levels in OKF6 cells were increased in response to PMA stimulation; moreover, the PMA-inducible expression of TGM2 was RIPK4-dependent (Fig. 6A). On the other hand, neither IRF6 (Fig. 6B) nor GRHL3 (Fig. 6C) were required for the stimulation of TGM2 gene expression by PMA. Similar to TGM1 (Fig. 5D), the PMAinducible expression of TGM2 was inhibited by CHX (Fig. 6D). These data suggest that RIPK4 functions independently of IRF6 and GRHL3 to regulate TGM2 gene expression in keratinocytes.

\section{Discussion}

Keratinocyte differentiation is central to maintaining the barrier functions of surface epithelia. Depending on anatomical location (e.g. gingiva and epidermis), keratinocytes may also become cornified, which increases their strength and hence barrier functions [2-4]. Underpinning cornification are transcriptional networks which regulate the expression of structural proteins and enzymes that mediate the formation of the cornified envelope. Herein, we have established a role for RIPK4 in regulating an IRF6-GRHL3-ELF3 transcriptional 
network that promotes the expression of small proline-rich proteins (e.g. SPRR1A) and transglutaminase-1 (TGM1) in keratinocytes. This would potentially position RIPK4 as an important regulator of keratinocyte cornification.

ELF3 was identified as a downstream target gene of RIPK4 signalling in OKF6 cells (e.g. oral keratinocytes) and epidermal keratinocytes. ELF3 is an epithelium-specific member of the ETS family of transcription factors [20,21], and has previously been demonstrated to be important for enterocyte differentiation [26]. RIPK4 promotes keratinocyte differentiation in part by phosphorylating and activating IRF6, which, in turn, induces the expression of additional transcriptional regulators (e.g. GRHL3) [12]. Our finding that the silencing of IRF6 inhibited the PMA-inducible expression of ELF3 therefore suggests that RIPK4 regulates ELF3 gene expression in keratinocytes, at least in part, by activating IRF6. The ELF3 gene does not appear to contain proximal IRF6 binding sites, and thus IRF6 likely regulates ELF3 gene expression indirectly, for example, by regulating the expression of an intermediary transcription factor.

In this regard, GRHL3 was demonstrated to be important for ELF3 gene expression in keratinocytes. GRHL3 has been shown to regulate epidermal keratinocyte differentiation by recruiting the Trithorax complex to the promoters of epidermal differentiation genes (e.g. involucrin) [27]. The upregulation of GRHL3 expression in OKF6 cells by PMA largely 308 preceded that of ELF3, and GRHL3 was demonstrated to be important for the PMA-inducible 309 expression of ELF3. ELF3 was recently reported to be a direct target gene of GRHL3 in 310 epidermal keratinocytes [28], and therefore GRHL3 likely functions downstream of RIPK4 311 and IRF6 to mediate ELF3 gene expression. Interestingly, ELF3 was suggested to function 312 upstream of GRHL3 in urothelial cells [29]. Additional studies will be required to fully 313 understand the functional relationship between GRHL3 and ELF3 in regulating epithelial cell 314 differentiation. 
Notably, inhibition of protein synthesis in OKF6 cells with cycloheximide resulted in transcription-dependent increases in IRF6, GRHL3, and ELF3 mRNA levels. This suggests that their expression in oral keratinocytes may also be regulated by transcriptional repressors. ZEB1, which is a key mediator of epithelial-mesenchymal transition [30], has been reported to repress IRF6 gene expression in mammary epithelial cells (e.g. MDA-MB-231 cells) [31]. However, whether ZEB1, or other repressors (e.g. SNAIL proteins), can regulate keratinocyte differentiation by suppressing IRF6, GRHL3, and ELF3 gene transcription is yet to be established.

The slower induction of ELF3 gene expression by PMA in OKF6 cells is consistent with ELF3 potentially playing a role during the later stages of oral keratinocyte differentiation; indeed, ELF3 has been shown to be most highly expressed in the most differentiated layers of the epidermis [32]. Importantly, ELF3 can regulate the expression of small proline-rich proteins (SPRRs) [21-23]. The cross-linking of SPRRs to other structural proteins (e.g. loricrin) increases the strength of the cornified envelope, while their relatively limited organised structure likely increases envelope elasticity [2, 4]. Consistently, we found that RIPK4 was important for the upregulation of SPRR1 gene expression in PMA-treated keratinocytes (e.g. OKF6 cells and epidermal keratinocytes). IRF6 and GRHL3 were also shown to be important for the upregulation of SPRR1 gene expression. Accordingly, RIPK4 likely regulates SPRR1 gene expression, at least in part, through its activation of an IRF6GRHL3-ELF3 transcriptional network.

The cross-linking of SPRRs, structural proteins (e.g. involucrin and loricrin), and lipids into the cornified envelope is catalysed by transglutaminases [25]. TGM1 in particular has 337 been shown to be essential for the formation of the cornified envelope and epidermal barrier 338 function [33]. In line with an earlier report demonstrating the direct regulation of TGM1 339 transcription by GRHL3 in epidermal keratinocytes [27], GRHL3 was shown here to be 
important for the upregulation of TGM1 gene expression in oral keratinocytes (e.g. OKF6 cells). RIPK4 and IRF6 were additionally shown to be important for the upregulation of TGM1 expression. Consequently, the ability of RIPK4 to activate the IRF6-GRHL3 pathway [12], and thereby induce TGM1 expression, potentially positions RIPK4 as a key regulator of keratinocyte cornification (Fig. 7).

RIPK4 was found to also be important for the upregulation of TGM2 gene expression in PMA-treated OKF6 cells. In contrast to its regulation of TGM1, RIPK4 regulated TGM2 expression independently of both IRF6 and GRHL3. TGM2 gene expression has previously been shown to be regulated by NF-кB $[34,35]$, and thus RIPK4 likely regulates TGM2 gene expression in keratinocytes via its ability to activate NF- $\mathrm{KB}$ [10-12]. Although TGM2 does not appear to play a role in the formation of the cornified envelope, its cross-linking of extracellular matrix $(\mathrm{ECM})$ components may nonetheless contribute to epithelial barrier function by decreasing ECM degradation as well as stabilising ECM-cell interactions [25]. Interestingly, RIPK4 has been found to be mutated in head and neck squamous cell carcinoma, including oral carcinoma [36], while the loss of ELF3 protein expression in oral squamous cell carcinoma was recently reported [37]. Therefore, the regulatory relationship between RIPK4 and ELF3 identified in this study may also be relevant to the development of squamous cell carcinoma.

\section{5. Conclusions}

360 In summary, we have identified and mechanistically defined a previously unrecognised 361 regulatory relationship between RIPK4 and ELF3 in keratinocytes. Thus, RIPK4 might 362 regulate the barrier functions of surface epithelia, at least in part, through its regulation of a 363 hierarchal IRF6-GRHL3-ELF3 transcription factor pathway. 


\section{Acknowledgements}

1

4

5367 Innovation and Science, and National Health and Medical Research Council Project Grant

7368628769.

8

9

10 


\section{References}

[1] K.C. Madison, Barrier function of the skin: "la raison d'etre" of the epidermis, J. Invest. Dermatol. 121 (2003) 231-241.

[2] R.B. Presland, B.A. Dale, Epithelial structural proteins of the skin and oral cavity: function in health and disease, Crit. Rev. Oral Biol. Medicine 11 (2000) 383-408.

[3] G. Donati, F.M. Watt, Stem cell heterogeneity and plasticity in epithelia, Cell Stem Cell 16 (2015) 465-476.

[4] E. Candi, R. Schmidt, G. Melino, The cornified envelope: a model of cell death in the skin, Nat. Rev. Mol. Cell Biol. 6 (2005) 328-340.

[5] M. Pasparakis, I. Haase, F.O. Nestle, Mechanisms regulating skin immunity and inflammation, Nat. Rev. Immunol. 14 (2014) 289-301.

[6] P. Holland, C. Willis, S. Kanaly, M. Glaccum, A. Warren, K. Charrier, J. Murison, J. Derry, G. Virca, T. Bird, J. Peschon, RIP4 is an ankyrin repeat-containing kinase essential for keratinocyte differentiation, Curr. Biol. 12 (2002) 1424-1428.

[7] P. De Groote, H.T. Tran, M. Fransen, G. Tanghe, C. Urwyler, B. De Craene, K. Leurs, B. Gilbert, G. Van Imschoot, R. De Rycke, C.J. Guerin, P. Holland, G. Berx, P. Vandenabeele, S. Lippens, K. Vleminckx, W. Declercq, A novel RIPK4-IRF6 connection is required to prevent epithelial fusions characteristic for popliteal pterygium syndromes, Cell Death Differ. 22 (2015) 1012-1024.

[8] E. Kalay, O. Sezgin, V. Chellappa, M. Mutlu, H. Morsy, H. Kayserili, E. Kreiger, A. Cansu, B. Toraman, E.M. Abdalla, Y. Aslan, S. Pillai, N.A. Akarsu, Mutations in RIPK4 cause the autosomal-recessive form of popliteal pterygium syndrome, Am. J. Hum. Genet. 90 (2012) 76-85.

[9] K. Mitchell, J. O'Sullivan, C. Missero, E. Blair, R. Richardson, B. Anderson, D. Antonini, J.C. Murray, A.L. Shanske, B.C. Schutte, R.A. Romano, S. Sinha, S.S. 
Bhaskar, G.C. Black, J. Dixon, M.J. Dixon, Exome sequence identifies RIPK4 as the Bartsocas-Papas syndrome locus, Am. J. Hum. Genet. 90 (2012) 69-75.

[10] E. Meylan, F. Martinon, M. Thome, M. Gschwendt, J. Tschopp, RIP4 (DIK/PKK), a novel member of the RIP kinase family, activates NF-kappa B and is processed during apoptosis, EMBO Rep. 3 (2002) 1201-1208.

[11] A. Muto, J. Ruland, L.M. McAllister-Lucas, P.C. Lucas, S. Yamaoka, F.F. Chen, A. Lin, T.W. Mak, G. Nunez, N. Inohara, Protein kinase C-associated kinase (PKK) mediates Bcl10-independent NF-kappa B activation induced by phorbol ester, J. Biol. Chem. 277 (2002) 31871-31876.

[12] M.Q. Kwa, J. Huynh, J. Aw, L. Zhang, T. Nguyen, E.C. Reynolds, M.J. Sweet, J.A. Hamilton, G.M. Scholz, Receptor-interacting protein kinase 4 and interferon regulatory factor 6 function as a signaling axis to regulate keratinocyte differentiation, J. Biol. Chem. 289 (2014) 31077-31087.

[13] M.Q. Kwa, J. Huynh, E.C. Reynolds, J.A. Hamilton, G.M. Scholz, Disease-associated mutations in IRF6 and RIPK4 dysregulate their signalling functions, Cell. Signal. 27 (2015) 1509-1516.

[14] M. Pasparakis, Regulation of tissue homeostasis by NF-kappaB signalling: implications for inflammatory diseases, Nat. Rev. Immunol. 9 (2009) 778-788.

[15] C.R. Ingraham, A. Kinoshita, S. Kondo, B. Yang, S. Sajan, K.J. Trout, M.I. Malik, M. Dunnwald, S.L. Goudy, M. Lovett, J.C. Murray, B.C. Schutte, Abnormal skin, limb and craniofacial morphogenesis in mice deficient for interferon regulatory factor 6 (Irf6), Nat Genet

Nature Genetics 38 (2006) 1335-1340. 
[16] R.J. Richardson, J. Dixon, S. Malhotra, M.J. Hardman, L. Knowles, R.P. BootHandford, P. Shore, A. Whitmarsh, M.J. Dixon, Irf6 is a key determinant of the keratinocyte proliferation-differentiation switch, Nat. Genet. 38 (2006) 1329-1334.

[17] E. Botti, G. Spallone, F. Moretti, B. Marinari, V. Pinetti, S. Galanti, P.D. De Meo, F. De Nicola, F. Ganci, T. Castrignano, G. Pesole, S. Chimenti, L. Guerrini, M. Fanciulli, G. Blandino, M. Karin, A. Costanzo, Developmental factor IRF6 exhibits tumor suppressor activity in squamous cell carcinomas, Proc. Natl. Acad. Sci. U. S. A. 108 (2011) 1371013715.

[18] G. de la Garza, J.R. Schleiffarth, M. Dunnwald, A. Mankad, J.L. Weirather, G. Bonde, S. Butcher, T.A. Mansour, Y.A. Kousa, C.F. Fukazawa, D.W. Houston, J.R. Manak, B.C. Schutte, D.S. Wagner, R.A. Cornell, Interferon regulatory factor 6 promotes differentiation of the periderm by activating expression of Grainyhead-like 3, J. Invest. Dermatol. 133 (2013) 68-77.

[19] M.Q. Kwa, G.M. Scholz, E.C. Reynolds, RIPK4 activates an IRF6-mediated proinflammatory cytokine response in keratinocytes, Cytokine 83 (2016) 19-26.

[20] M.J. Tymms, A.Y. Ng, R.S. Thomas, B.C. Schutte, J. Zhou, H.J. Eyre, G.R. Sutherland, A. Seth, M. Rosenberg, T. Papas, C. Debouck, I. Kola, A novel epithelial-expressed ETS gene, ELF3: human and murine cDNA sequences, murine genomic organization, human mapping to 1q32.2 and expression in tissues and cancer, Oncogene 15 (1997) 2449-2462.

[21] P. Oettgen, R.M. Alani, M.A. Barcinski, L. Brown, Y. Akbarali, J. Boltax, C. Kunsch, K. Munger, T.A. Libermann, Isolation and characterization of a novel epitheliumspecific transcription factor, ESE-1, a member of the ets family, Mol. Cell. Biol. 17 (1997) 4419-4433. 
[22] M.W. Sark, D.F. Fischer, E. de Meijer, P. van de Putte, C. Backendorf, AP-1 and ets transcription factors regulate the expression of the human SPRR1A keratinocyte terminal differentiation marker, J. Biol. Chem. 273 (1998) 24683-24692.

[23] S.P. Reddy, H. Vuong, P. Adiseshaiah, Interplay between proximal and distal promoter elements is required for squamous differentiation marker induction in the bronchial epithelium: role for ESE-1, Sp1, and AP-1 proteins, J. Biol. Chem. 278 (2003) 21378 21387.

[24] C.H. Lee, L.N. Marekov, S. Kim, J.S. Brahim, M.H. Park, P.M. Steinert, Small prolinerich protein 1 is the major component of the cell envelope of normal human oral keratinocytes, FEBS Lett. 477 (2000) 268-272.

[25] L. Lorand, R.M. Graham, Transglutaminases: crosslinking enzymes with pleiotropic functions, Nat. Rev. Mol. Cell Biol. 4 (2003) 140-156.

[26] A.Y. Ng, P. Waring, S. Ristevski, C. Wang, T. Wilson, M. Pritchard, P. Hertzog, I. Kola, Inactivation of the transcription factor Elf3 in mice results in dysmorphogenesis and altered differentiation of intestinal epithelium, Gastroenterology 122 (2002) 14551466.

[27] A.S. Hopkin, W. Gordon, R.H. Klein, F. Espitia, K. Daily, M. Zeller, P. Baldi, B. Andersen, GRHL3/GET1 and trithorax group members collaborate to activate the epidermal progenitor differentiation program, PLoS Genet. 8 (2012) e1002829.

[28] W.M. Gordon, M.D. Zeller, R.H. Klein, W.R. Swindell, H. Ho, F. Espetia, J.E. Gudjonsson, P.F. Baldi, B. Andersen, A GRHL3-regulated repair pathway suppresses immune-mediated epidermal hyperplasia, J. Clin. Invest. 124 (2014) 5205-5218.

[29] M. Bock, J. Hinley, C. Schmitt, T. Wahlicht, S. Kramer, J. Southgate, Identification of ELF3 as an early transcriptional regulator of human urothelium, Dev. Biol. 386 (2014) 321-330. 
[30] C. Vandewalle, F. Van Roy, G. Berx, The role of the ZEB family of transcription factors in development and disease, Cell. Mol. Life Sci. 66 (2009) 773-787.

[31] E. Bonnet, M. Tatari, A. Joshi, T. Michoel, K. Marchal, G. Berx, Y. Van de Peer, Module network inference from a cancer gene expression data set identifies microRNA regulated modules, PLoS One 5 (2010) e10162.

[32] J.M. Andreoli, S.I. Jang, E. Chung, C.M. Coticchia, P.M. Steinert, N.G. Markova, The expression of a novel, epithelium-specific ets transcription factor is restricted to the most differentiated layers in the epidermis, Nucleic Acids Res. 25 (1997) 4287-4295.

[33] M. Matsuki, F. Yamashita, A. Ishida-Yamamoto, K. Yamada, C. Kinoshita, S. Fushiki, E. Ueda, Y. Morishima, K. Tabata, H. Yasuno, M. Hashida, H. Iizuka, M. Ikawa, M. Okabe, G. Kondoh, T. Kinoshita, J. Takeda, K. Yamanishi, Defective stratum corneum and early neonatal death in mice lacking the gene for transglutaminase 1 (keratinocyte transglutaminase), Proc. Natl. Acad. Sci. U. S. A. 95 (1998) 1044-1049.

[34] A. Mirza, S.L. Liu, E. Frizell, J. Zhu, S. Maddukuri, J. Martinez, P. Davies, R. Schwarting, P. Norton, M.A. Zern, A role for tissue transglutaminase in hepatic injury and fibrogenesis, and its regulation by NF-kappaB, Am. J. Physiol. 272 (1997) G281288.

[35] L. Ai, R.R. Skehan, J. Saydi, T. Lin, K.D. Brown, Ataxia-Telangiectasia, Mutated (ATM)/Nuclear Factor kappa light chain enhancer of activated B cells (NFkappaB) signaling controls basal and DNA damage-induced transglutaminase 2 expression, J. Biol. Chem. 287 (2012) 18330-18341.

[36] N. Stransky, A.M. Egloff, A.D. Tward, A.D. Kostic, K. Cibulskis, A. Sivachenko, G.V. Kryukov, M.S. Lawrence, C. Sougnez, A. McKenna, E. Shefler, A.H. Ramos, P. Stojanov, S.L. Carter, D. Voet, M.L. Cortes, D. Auclair, M.F. Berger, G. Saksena, C. Guiducci, R.C. Onofrio, M. Parkin, M. Romkes, J.L. Weissfeld, R.R. Seethala, L. 
Wang, C. Rangel-Escareno, J.C. Fernandez-Lopez, A. Hidalgo-Miranda, J. Melendez-

Zajgla, W. Winckler, K. Ardlie, S.B. Gabriel, M. Meyerson, E.S. Lander, G. Getz, T.R.

Golub, L.A. Garraway, J.R. Grandis, The mutational landscape of head and neck squamous cell carcinoma, Science 333 (2011) 1157-1160.

[37] A.A. AbdulMajeed, A.J. Dalley, C.S. Farah, Loss of ELF3 immunoexpression is useful for detecting oral squamous cell carcinoma but not for distinguishing between grades of epithelial dysplasia, Ann Diagn Pathol 17 (2013) 331-340. 


\section{Figure Legends}

Fig. 1. RIPK4 and IRF6 dependent regulation of ELF3 gene expression in human

keratinocytes. (A-D) OKF6 cells were transfected with a (A-B) RIPK4 or (C-D) IRF6 siRNA $(+)$, or control siRNA (-). (A) RIPK4 and (C) IRF6 mRNA levels in cells transfected with the control siRNA were each given an arbitrary value of $100 \%(n=3)$. (B and D) The cells were subsequently stimulated with PMA for $6 \mathrm{~h}$, and ELF3 mRNA levels measured $(n=3)$. (E-F) Human epidermal keratinocytes were transfected with a (E) RIPK4 or (F) IRF6 siRNA (+), or control siRNA (-). The cells were subsequently stimulated with PMA for $6 \mathrm{~h}$, and ELF3 mRNA levels measured $(n=3) . * * p<0.01 ; * p<0.05$.

Fig. 2. GRHL3 dependent regulation of ELF3 gene expression in human keratinocytes. (A-B) OKF6 cells were stimulated with PMA for the times indicated, and (A) ELF3 and (B) GRHL3 mRNA levels measured $(n=3)$. (C) OKF6 cells were pretreated with actinomycin D (ActD) for 30 min, stimulated with PMA for $6 \mathrm{~h}$, and ELF3 mRNA levels measured $(n=4)$. (D-F) OKF6 cells were transfected with a GRHL3 (+) or control $(-)$ siRNA. The cells were subsequently stimulated with PMA for $24 \mathrm{~h}$ (D and E) or $2 \mathrm{~h}(\mathrm{~F})$, and (D) ELF3, (E) IVL, and (F) OVOL1 mRNA levels measured $(n=3)$. ${ }^{* *} p<0.01 ; * p<0.05$.

Fig. 3. Inhibition of protein synthesis results in increased ELF3 gene expression in human keratinocytes. OKF6 cells were pretreated with cycloheximide (CHX) and/or actinomycin D (ActD) for 30 min, stimulated with PMA for $6 \mathrm{~h}$, and (A) ELF3, (B) IRF6, (C)

GRHL3, and (D) RIPK4 mRNA levels measured by qPCR $(n=5)$. ** $p<0.01 ; * p<0.05$.

Fig. 4. RIPK4 and IRF6 dependent regulation of SPRR1 gene expression in human keratinocytes. (A-B and E-H) OKF6 cells and (C-D) normal human epidermal keratinocytes 
were transfected with a (A-D) RIPK4, (E-F) IRF6, or (G-H) GRHL3 siRNA (+), or a control siRNA (-). The cells were subsequently stimulated with PMA for $24 \mathrm{~h}$, and (A, C, E, and G) SPRR1A and (B, D, F, and H) SPRR1B mRNA levels measured ( $n=4)$. (I-J) OKF6 cells were pretreated with cycloheximide (CHX) for $30 \mathrm{~min}$, stimulated with PMA for $6 \mathrm{~h}$, and (I) SPRR1A and (J) SPRR1B mRNA levels measured $(n=4)$. ** $p<0.01 ; * p<0.05$.

\section{Fig. 5. RIPK4 and IRF6 dependent regulation of TGM1 gene expression in human} keratinocytes. (A, C, and D) OKF6 cells and (B) normal human epidermal keratinocytes were transfected with a (A-B) RIPK4, (C) IRF6, or (D) GRHL3 siRNA (+), or a control siRNA (-). The cells were subsequently stimulated with PMA for $24 \mathrm{~h}$, and TGM1 mRNA levels measured $(n=3-5)$. (E) OKF6 cells were pretreated with cycloheximide (CHX) for 30 min, stimulated with PMA for $6 \mathrm{~h}$, and TGM1 mRNA levels measured $(n=5)$. ${ }^{* *} p<0.01$; * $p<0.05$.

Fig. 6. RIPK4 dependent regulation of TGM2 gene expression in human keratinocytes. (A-C) OKF6 cells were transfected with a (A) RIPK4, (B) IRF6, or (C) GRHL3 siRNA (+), or a control siRNA (-). The cells were subsequently stimulated with PMA for $24 \mathrm{~h}$, and TGM2 mRNA levels measured $(n=3)$. (D) OKF6 cells were pretreated with cycloheximide (CHX) for $30 \mathrm{~min}$, stimulated with PMA for $6 \mathrm{~h}$, and TGM2 mRNA levels measured $(n=5)$. $* * p<0.01$.

\section{Fig. 7. A proposed model for the regulation of ELF3 gene expression in human}

keratinocytes by RIPK4. The activation (phosphorylation) of IRF6 by RIPK4 promotes the expression of GRHL3, which induces, either directly or indirectly, ELF3 gene expression, culminating in the upregulation of SPRR1 gene expression. The upregulation of GRHL3 
550 expression by RIPK4 and IRF6 concomitantly promotes the expression of TGM1, an enzyme

1

2551 that catalyses the cross-linking of small proline-rich proteins (e.g. SPRR1) to other structural

4

5552 proteins to facilitate the formation of the cornified envelope of terminally differentiated

6

7553 keratinocytes. RIPK4 also regulates, independently of IRF6 and GRHL3, the expression of

554 TGM2. 
Figure 1

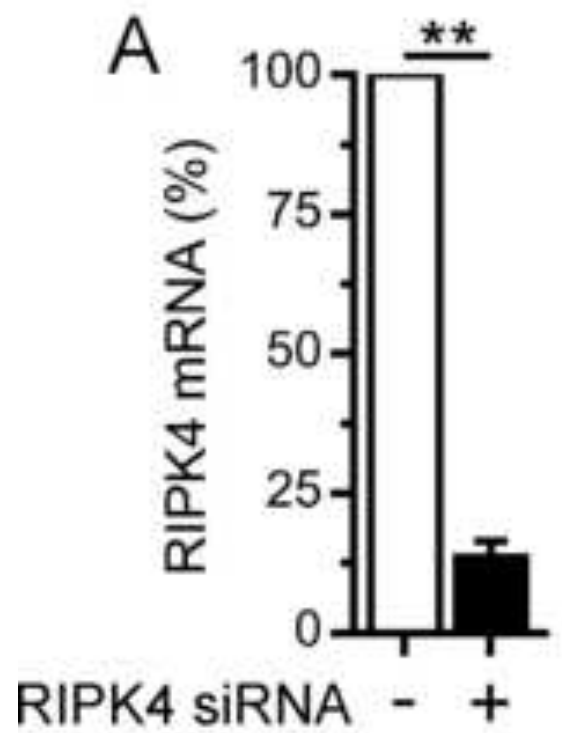

B
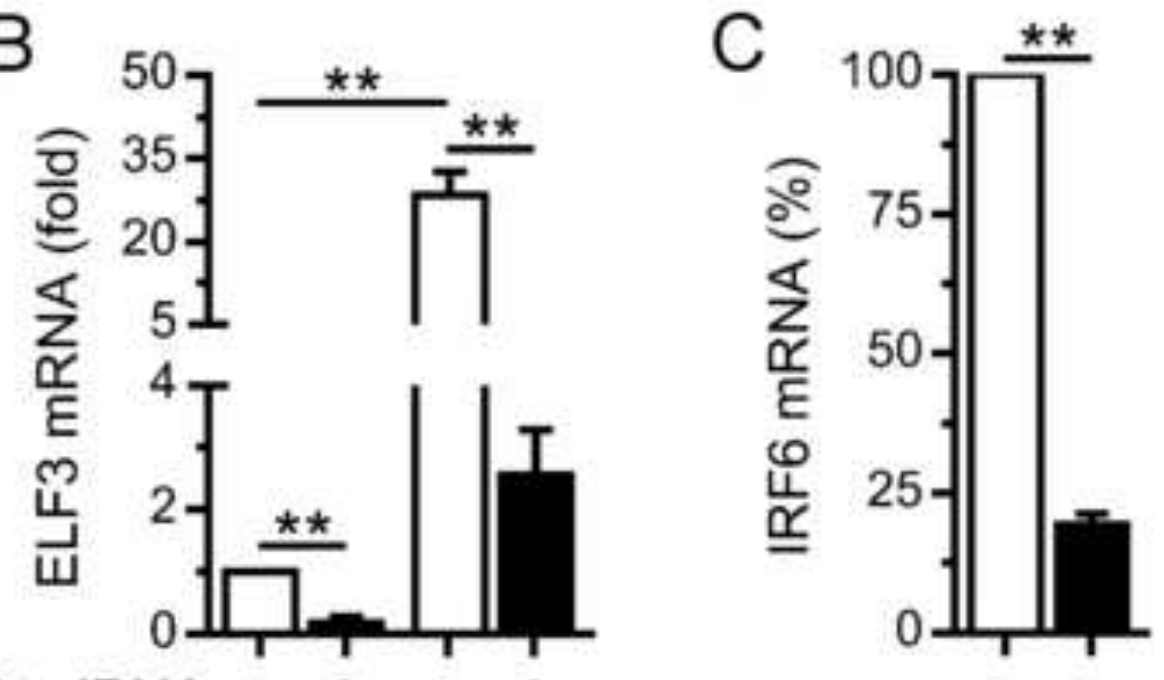

RIPK4 siRNA - +

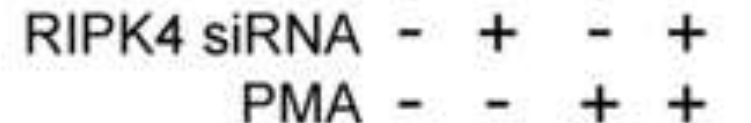

IRF6 siRNA - +

D

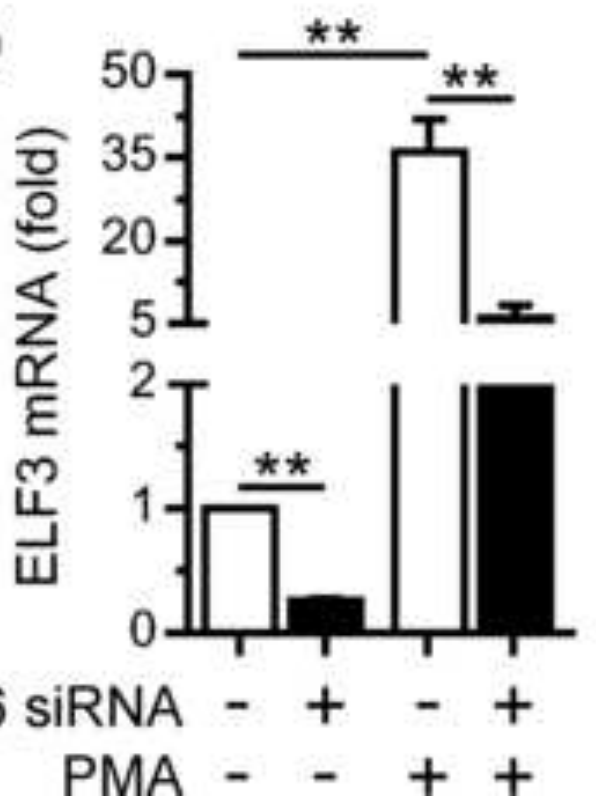

E

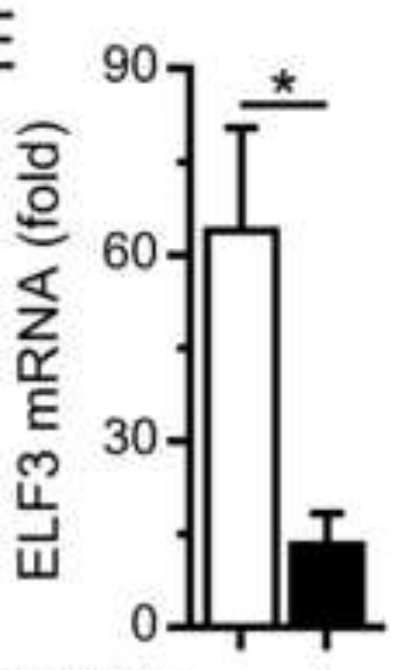

RIPK4 SIRNA - +
F

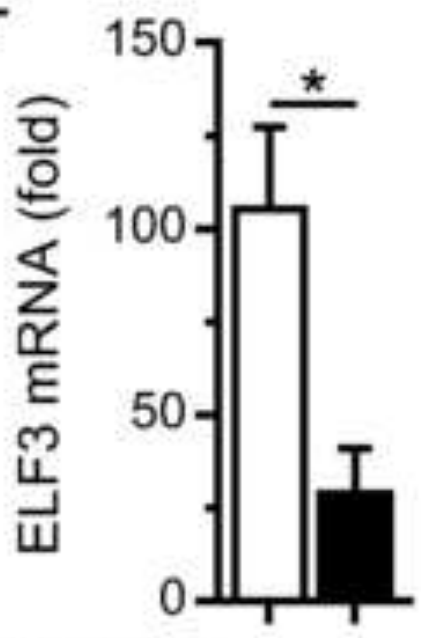

$\mathrm{PMA}++$

IRF6 siRNA - + ++
IRF6 siRNA - +

$\mathrm{PMA}++$ 
Figure 2
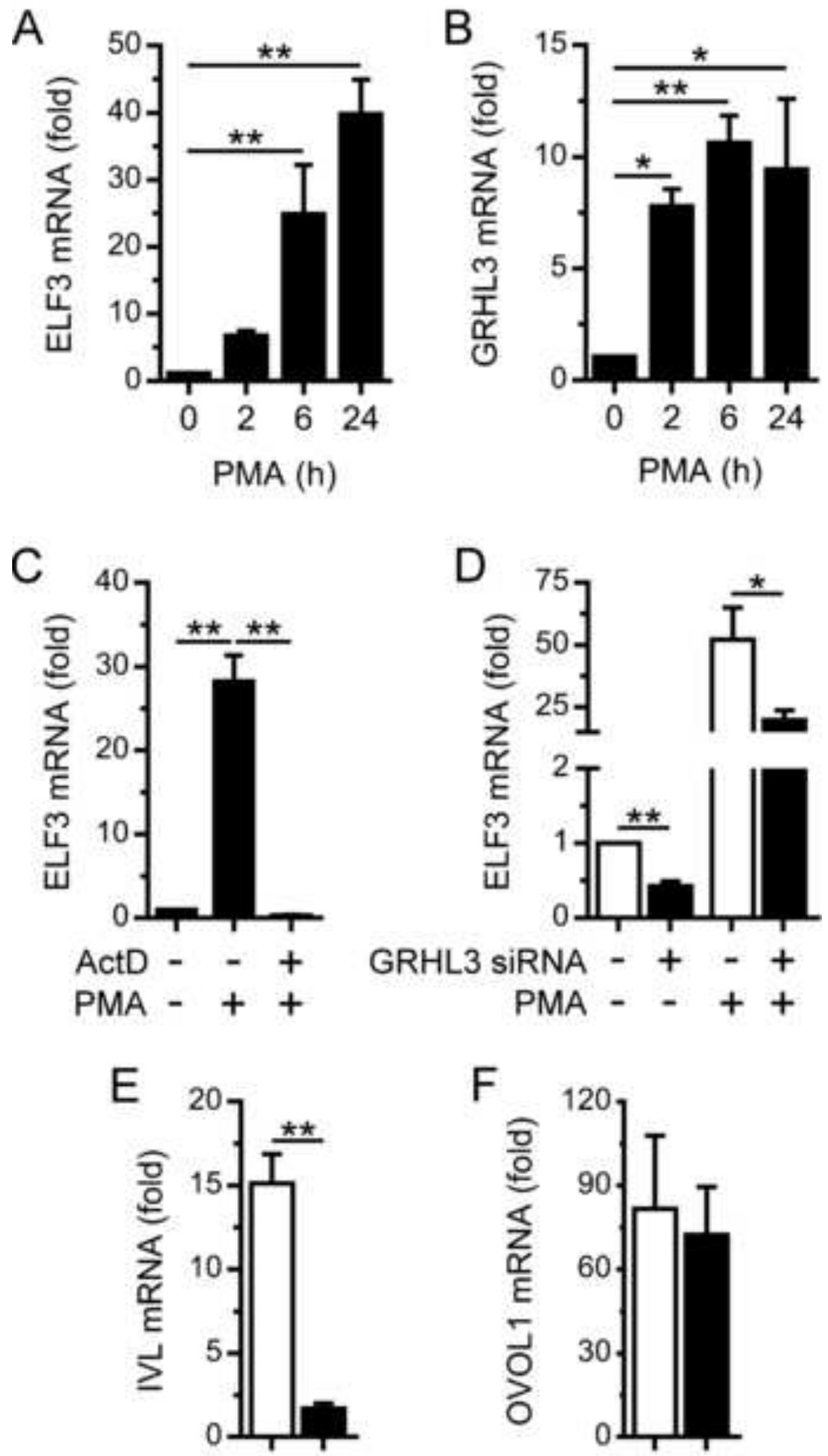

GRHL3 SIRNA - +

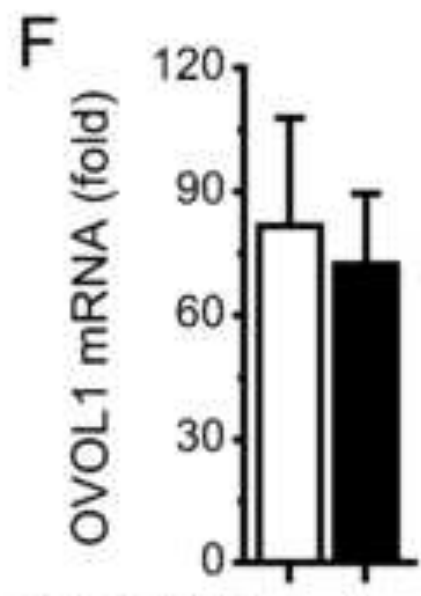

$$
\begin{array}{rr}
\text { siRNA - + } & \text { GRHL3 SIRNA - + } \\
\text { PMA + }+ & \text { PMA }++
\end{array}
$$


Figure 3
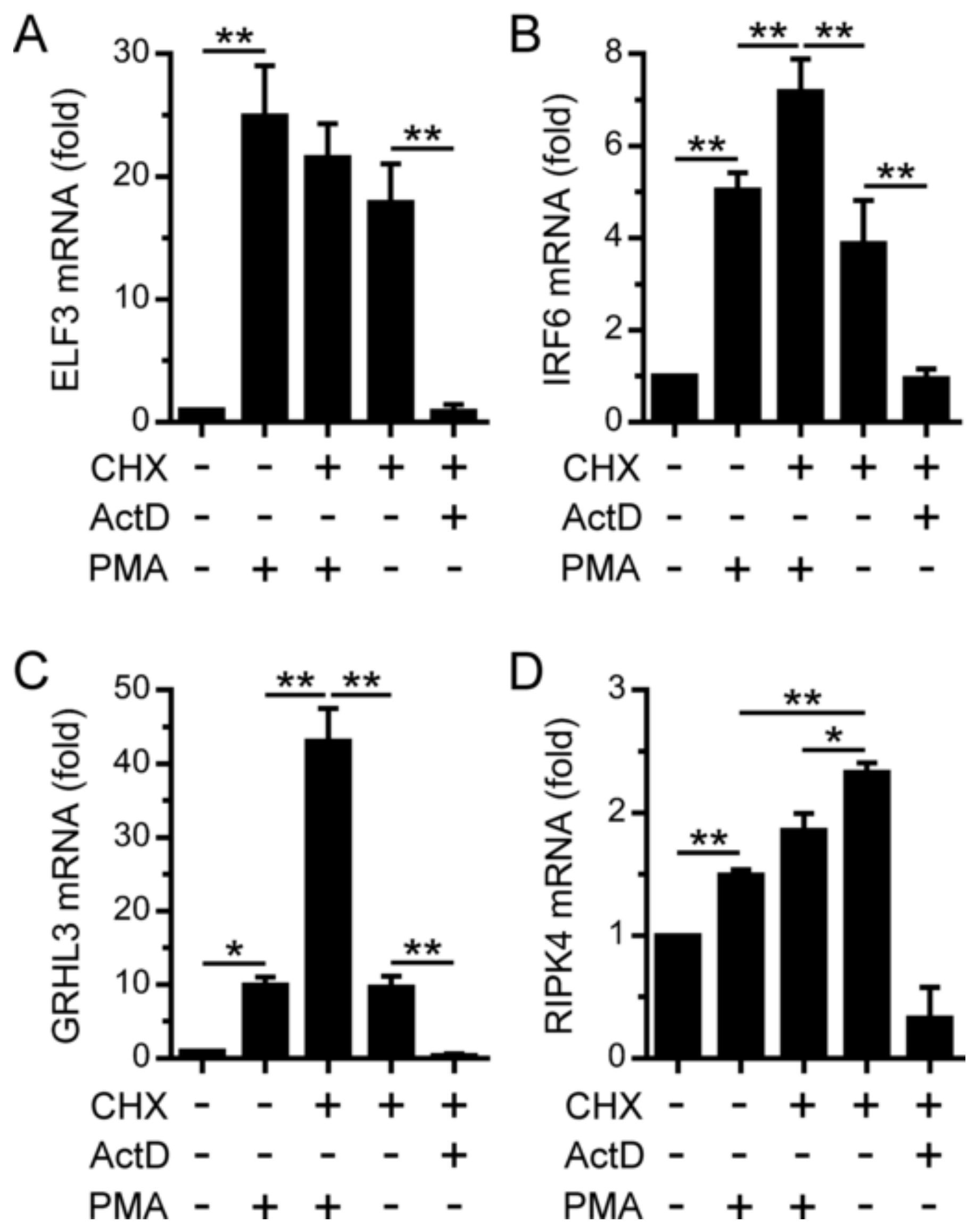
Figure 4
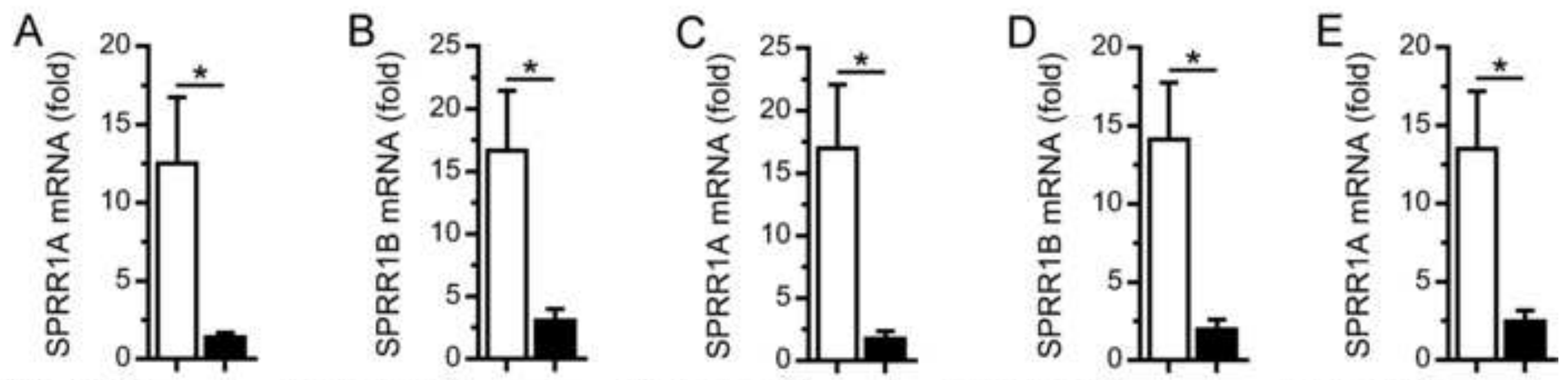

RIPK4 siRNA - + RIPK4 siRNA - + RIPK4 siRNA - + RIPK4 siRNA - + IRF6 siRNA - +
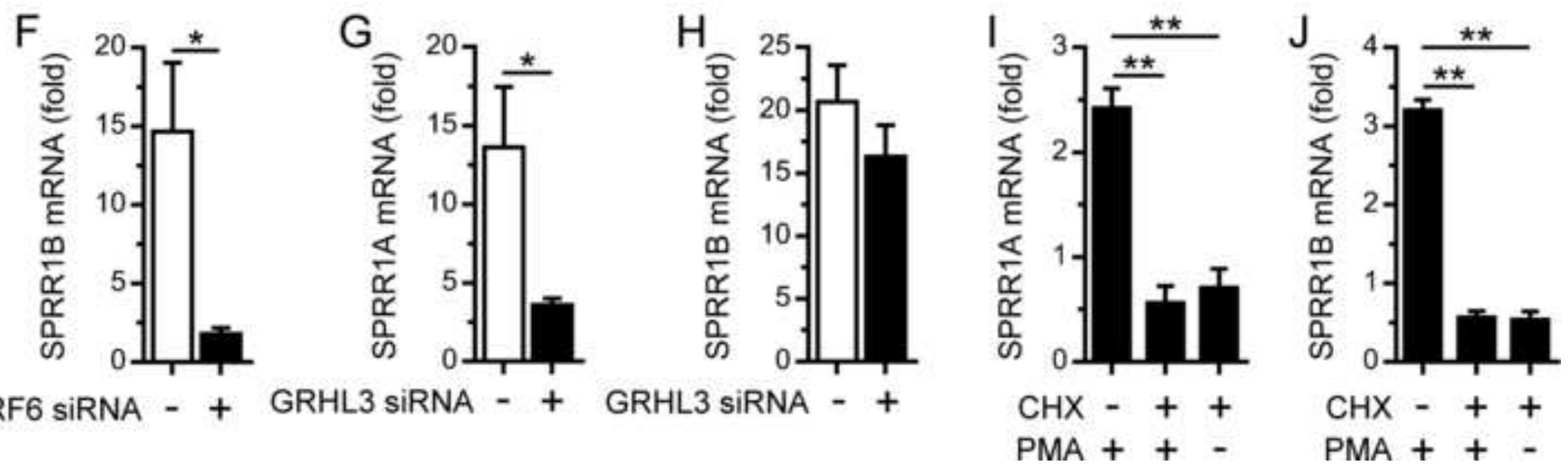

$\mathrm{PMA}++-$ 
Figure 5
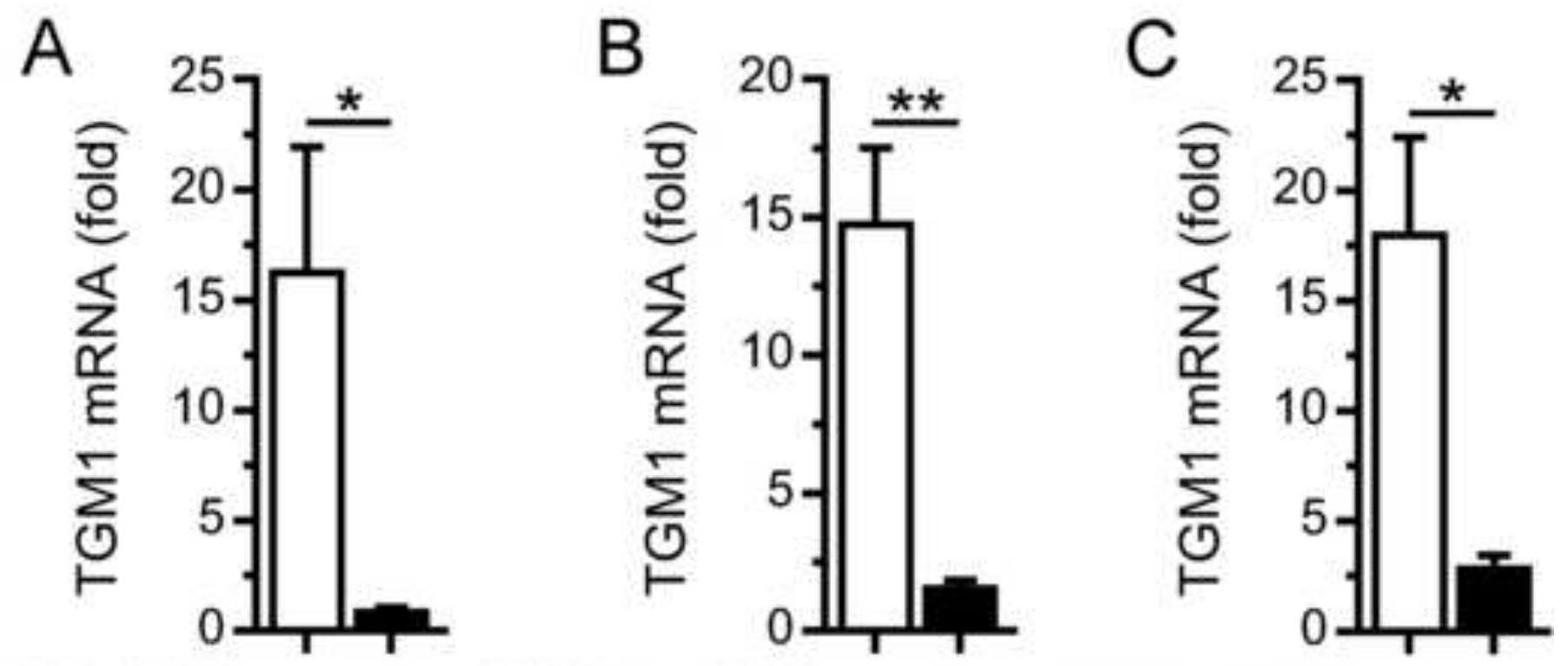

RIPK4 siRNA - + RIPK4 siRNA - + IRF6 siRNA - +
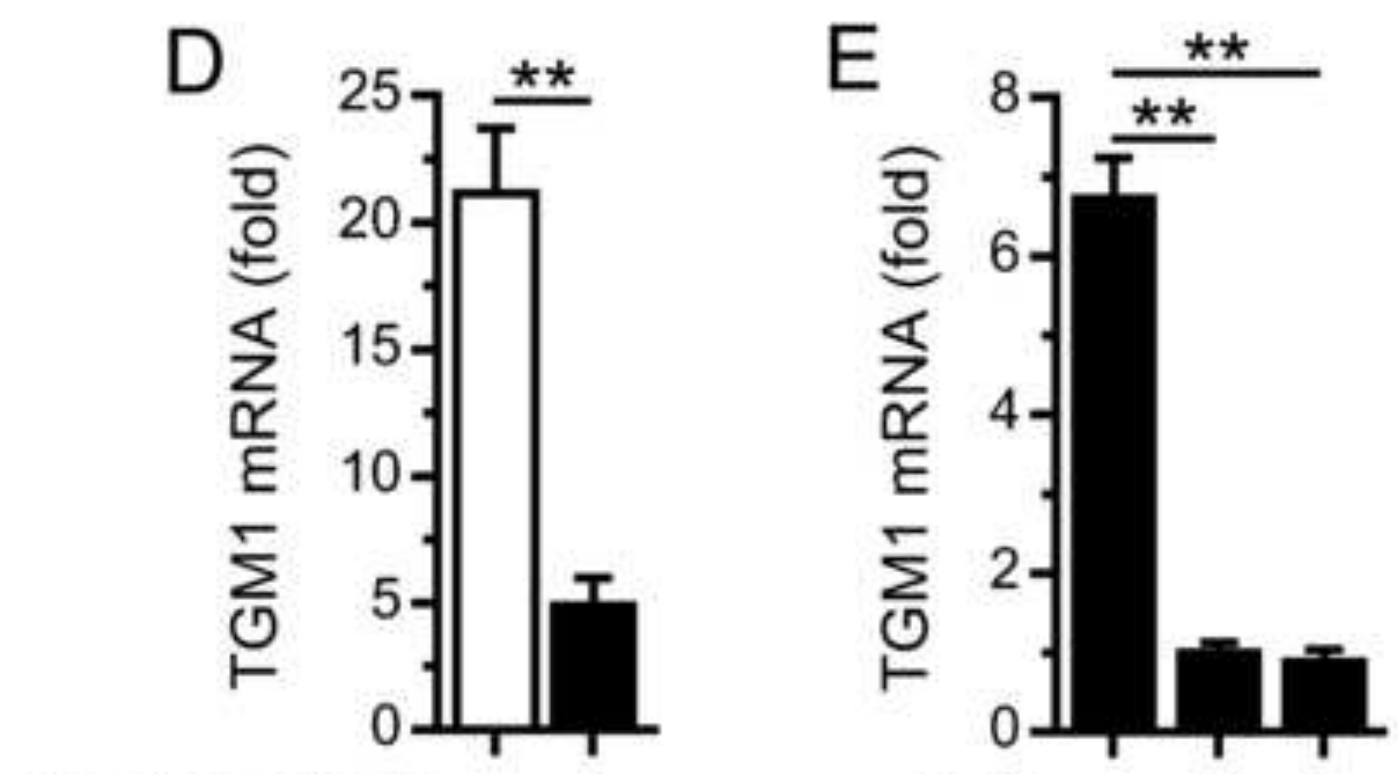

GRHL3 siRNA - +

$\mathrm{CHX}-++$

$\mathrm{PMA}++-$ 
Figure 6
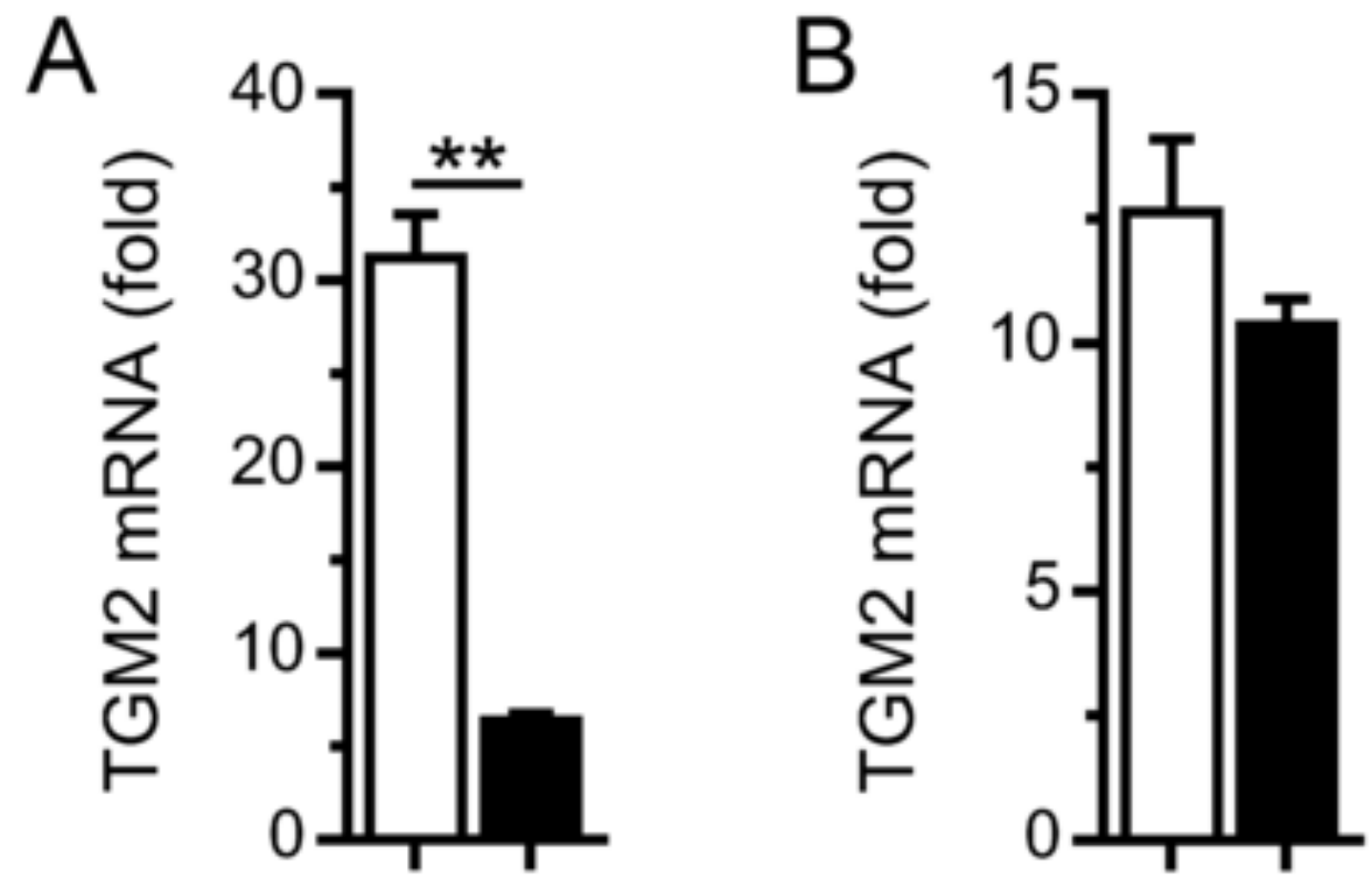

RIPK4 SiRNA - +

IRF6 siRNA - +

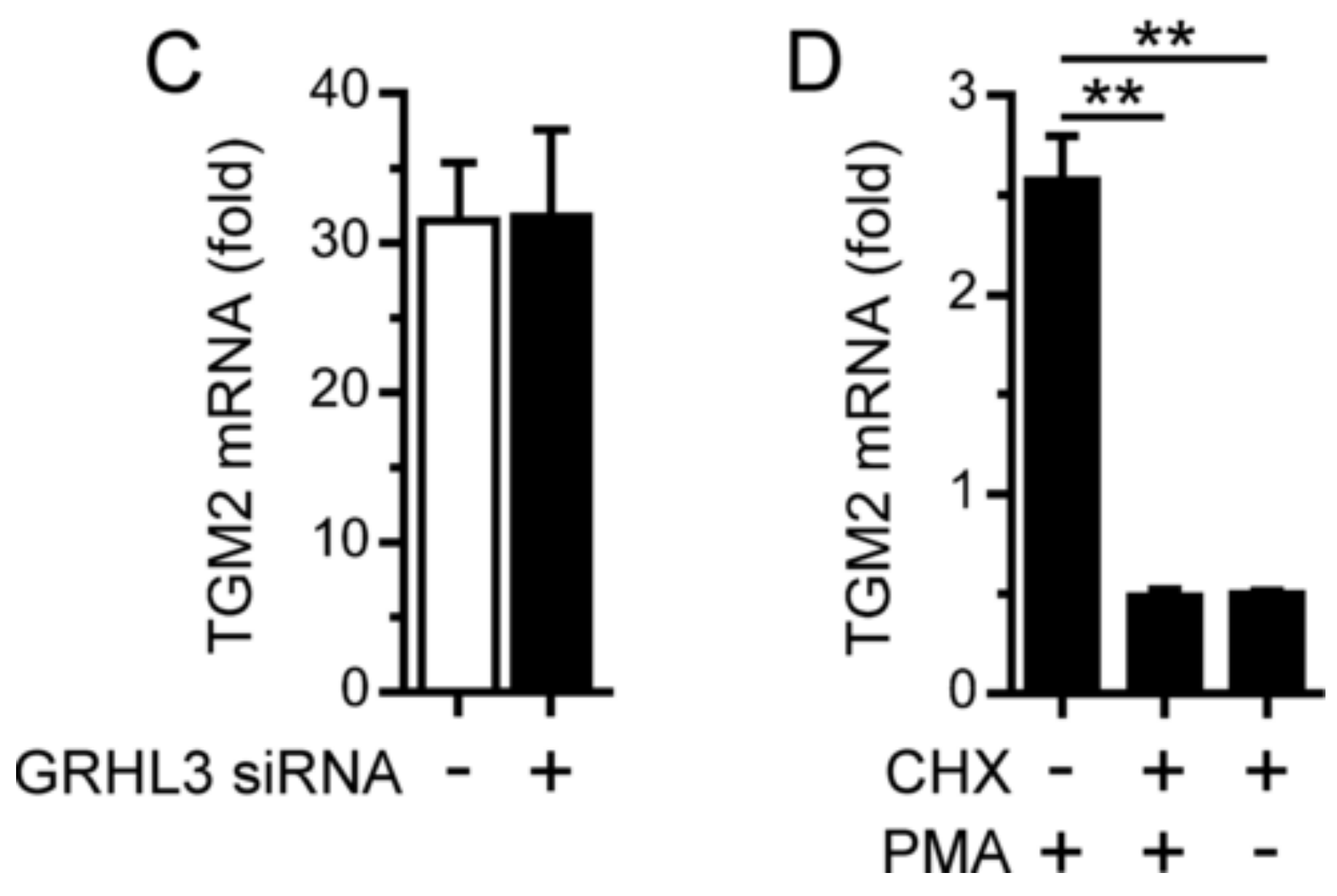


Figure 7
Click here to download high resolution image

Figure 7

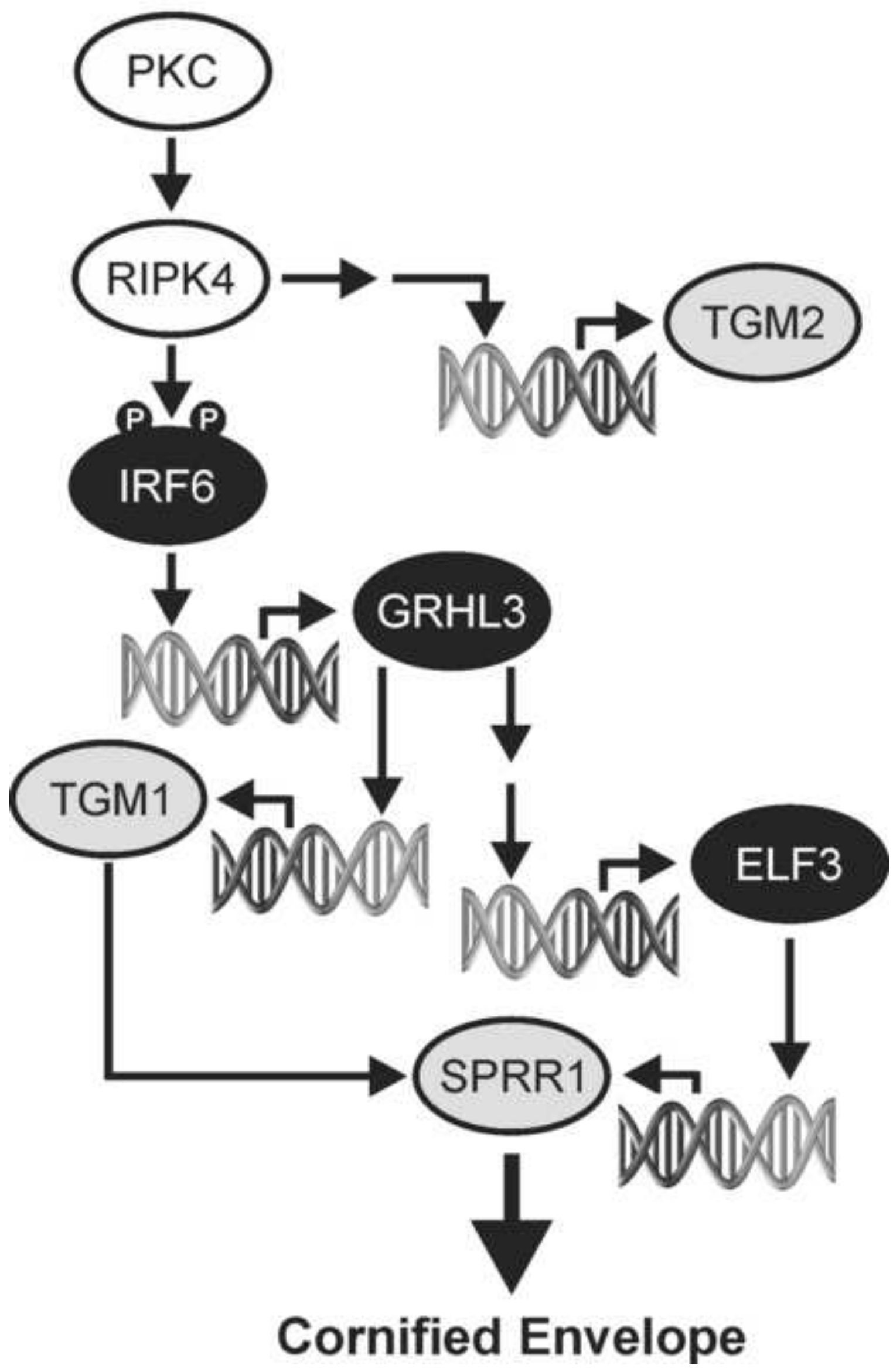




\section{University Library}

\section{- M M N E R VA A gateway to Melbourne's research publications}

Minerva Access is the Institutional Repository of The University of Melbourne

Author/s:

Scholz, GM;Sulaiman, NS;Al Baiiaty, S;Kwa, MQ;Reynolds, EC

Title:

A novel regulatory relationship between RIPK4 and ELF3 in keratinocytes

Date:

2016-12-01

Citation:

Scholz, G. M., Sulaiman, N. S., Al Baiiaty, S., Kwa, M. Q. \& Reynolds, E. C. (2016). A novel regulatory relationship between RIPK4 and ELF3 in keratinocytes. CELLULAR SIGNALLING, 28 (12), pp.1916-1922. https://doi.org/10.1016/j.cellsig.2016.09.006.

Persistent Link:

http://hdl.handle.net/11343/123327 